\title{
Exotic geophysical phenomena observed in an environmental neutron flux study using EAS PRISMA detectors
}

\author{
Victor Alekseenko ${ }^{1}$, Anastasia Bagrova ${ }^{1,2}$, Shuwang Cui ${ }^{3}$ a , Yayun $\mathrm{He}^{3}$, Bingbing $\mathrm{Li}^{3}, \mathrm{Xinhua} \mathrm{Ma}^{4}, \mathrm{Egor} \mathrm{Pozdnyakov}^{5}$, \\ Oleg Shchegolev ${ }^{1}$, Yuri Stenkin ${ }^{1,2}$, and Vladimir Stepanov ${ }^{1}$ \\ 1 Institute for Nuclear Research, Russian Academy of Sciences, Russia \\ 2 Nuclear Research National University "Moscow Engineering Physics Institute”, Russia \\ 3 The College of Physics and Information Engineering, Hebei Normal University, China \\ ${ }^{4}$ Key Laboratory of Particle Astrophysics, Institute of High Energy Physics, Chinese Academy of Sciences, China \\ 5 CJSC NPF “Luminofor”, Russia
}

\begin{abstract}
Some exotic geophysical events are observed by a global net of electron-neutron detectors (en-detectors) developed in the framework of the PRISMA EAS project. Our en-detectors running both on the Earth's surface and underground are continuously measuring the environmental thermal neutron flux. Thermal neutrons are in equilibrium with media and are therefore sensitive to many geophysical phenomena, which are exotic for people studying ultra high-energy cosmic rays or carrying out low background experiments deep underground.
\end{abstract}

\section{Introduction}

The en-detectors were developed for Extensive Air Shower (EAS) experiments in the framework of the PRISMA project [1]. The main issue of the project is the possibility of the EAS array to record secondary thermal neutrons accompanying EAS. The project will be realized in a few years in collaboration with the LHAASO project as the PRISMA-LHAASO array. One of the PRISMA-LHAASO aims is to monitor the environmental thermal neutron flux to study variations of possible neutron background change. We have found some interesting phenomena in the neutron flux having geophysical or Sun-Earth origin. They are: rain and snow effect, Forbush effect, daily temperature variations, seasonal and moon tidal waves, barometric pumping effect, etc.

\section{En-detector}

A novel type of thermal neutron scintillation detector (en-detector) based on a well-known specialized solid granulated alloy, ${ }^{6} \mathrm{LiF}+\mathrm{ZnS}(\mathrm{Ag})$, is used to detect thermal and epithermal neutrons. The thickness of the scintillator layer is $\sim 30 \mathrm{mg} / \mathrm{cm}^{2}$. The sensitive area of the scintillator surface is $0.75 \mathrm{~m}^{2}$. A schematic view of the en-detector and its performances can be found elsewhere [2]. The detection efficiency for thermal neutrons was found to be $\sim 20 \%$. The efficiency displays a $\sim 1 / \mathrm{v}$ dependence on the neutron velocity $\mathrm{v}$, typical for thin detectors. Due to the thin scintillator layer, relativistic charged particles, such as electrons or muons, produce very small signals below the threshold. This allows us to use the detector in a scaler mode to study the variations in the thermal neutron flux. Due to the existence of several scintillating time constants

a e-mail: cui_shuwang@163.com in $\mathrm{ZnS}(\mathrm{Ag}$ ) (from $40 \mathrm{~ns}$ to several $\mu \mathrm{s}$ ), the pulse shape produced by heavy and slowly moving particles (alpha and triton emission after neutron capture on a ${ }^{6} \mathrm{Li}$ target) is longer than that produced by relativistic charged particles or by PMT's noise. Therefore, we exclude background signals applying a pulse shape selection method.

A distinctive feature of our data acquisition process is that all pulses from PMT are integrated with a time constant of $5 \mu \mathrm{s}$ and then digitized by a FADC in a $100 \mu \mathrm{s}$ time gate with a step of $50 \mathrm{~ns}$. In other words, on-line pulse shape analysis and selection are used to count real neutron pulses and to reject, though still counting, the noise pulses. The technical details and some preliminary results can be found elsewhere [2]. The en-detector used in this work is located at a depth of $25 \mathrm{hg} / \mathrm{cm}^{2}$ in a shaft of muon detectors of the EAS-MSU array (Moscow State University) [3]. The underground room where the detector has been running for $\sim 4$ years has no forced ventilation and has no protection against soil gas penetration. Experimental data were accumulated as a time series with $1 \mathrm{~min}$ steps. The detector has very stable longterm behavior, which is controlled through daily energy deposit spectra.

\section{Overview of the observed "exotic" phenomena in neutron flux}

As mentioned above, the en-detectors were developed for Extensive Air Shower studies in the framework of the PRISMA project. Studying environmental neutron backgrounds at different sites, we have observed some "exotic" effects. We have to emphasize here that the observed phenomena are exotic only for high-energy physicists but are well-known for geophysicists. 


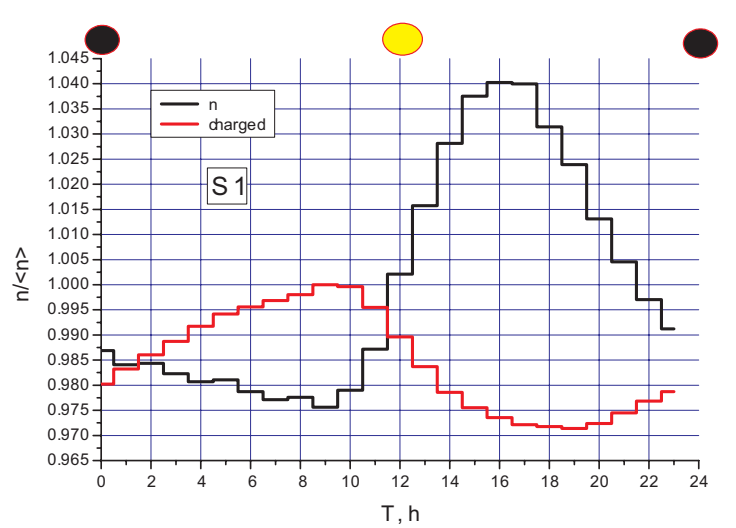

Figure 1. Diurnal wave $\mathrm{S} 1$ observed in PRISMA-YBJ en-detectors in local astronomical time. Circles shows sun culminations. No corrections were applied.

\subsection{Diurnal wave}

Four of our en-detectors have been running continuously in Tibet (Yangbajing, $4300 \mathrm{~m}$ a.s.1.) since August 2013 as the PRISMA-YBJ array [2] being a prototype of PRISMA. During this study a long data time series was accumulated and analyzed. Using the superimposed epoch method, the diurnal wave, S1, was obtained for both pulses selected as "neutron" and "charged" shown in Fig. 1. As one can see, the two types of events anti-correlate. This could be explained as follows:

i) Neutron wave is very close to the near surface air temperature wave because thermal neutrons are in equilibrium with the media and their mean velocity, $<\mathrm{v}>$, is proportional to $\operatorname{sqrt}(\mathrm{T})$ while the flux, $\mathrm{F}$, is $\sim \mathrm{C}<\mathrm{v}>$, where $\mathrm{C}$ is the neutron concentration in air;

ii) Pulses selected as "charged" are partially connected with decays of radon in air [3] and so they have not a temperature origin, and mostly follow the wave of radon exhalation from soil and its concentration in air.

\subsection{Moon tidal waves}

Using the superimposed epoch analysis for longer periods, we found some known lunar tidal periods, namely the lunar month $(\mathrm{T}=29.5 \mathrm{~d})$ and its 4th harmonic which is shown in Fig. 2. As one can see the wave is clearly observed with an amplitude of about $0.5 \%$. In addition, a delay of $\sim 1$ day between the syzygies and the flux maximum in neutrons can be seen, while that for "charged" is $\sim 1.5$ day. This phenomenon can be explained by the influence of Moon tidal forces on the Earth's crust resulting in a change in soil radon diffusion and thus neutron production in the upper soil level.

\subsection{Barometric pumping effect}

A 2-day delayed anti-correlation was observed in the underground en-detector situated at a depth of $25 \mathrm{~m}$ of water equivalent in the mine of the EAS-MSU array [4]. Cosmic ray hadrons do not penetrate to this depth and thus the neutron barometric coefficient should be close to zero. But, when the air pressure monotonously decreases for many days, all underground gases including radon

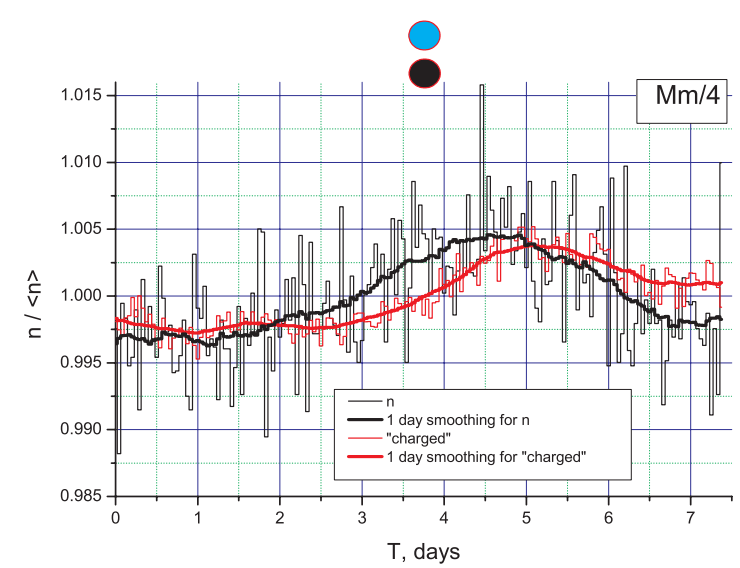

Figure 2. 4th harmonic of Moon month ( $\mathrm{T}=7.38 \mathrm{~d})$ observed at Tibet level. Positions of moon syzygies are marked by circles.
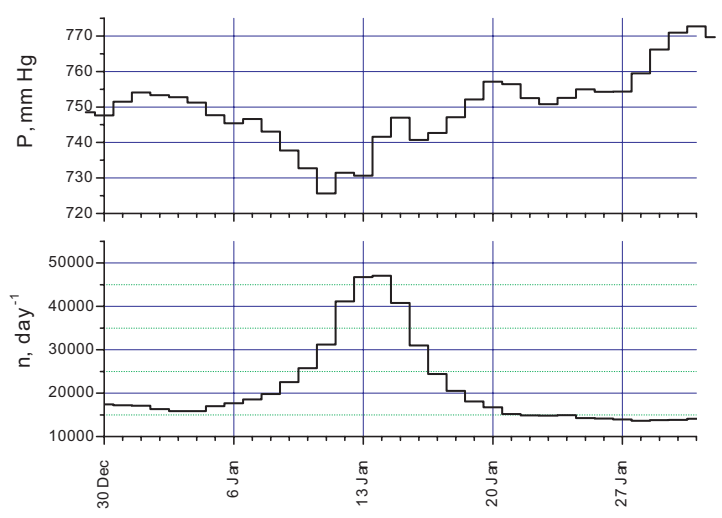

Figure 3. Anti-correlation between daily air pressure and normalized daily neutron counting rate in January, 2014.

(Rn-222) undergo the so called barometric pumping effect well known to geophysicists, when soil gases effectively diffuse from deeper to upper soil levels and thence to the atmosphere. We have found the barometric pumping effect exists not only for radon but also for thermal neutrons. The effect results in a spontaneous rise of the thermal neutron flux up to a factor of 3 . After applying the 2-day delay to the pressure data, we estimated the correlation between barometric pressure and neutron counts at the period when the biggest neutron increase was observed (Jan. 4 to Jan. 23, 2014). Anti-correlation between the absolute 2-day delayed mean daily air pressure, $\mathrm{P}$, and the normalized daily neutron counting rate, $n /\langle n>$, for this 19 day period is shown in Figs. 3, 4. As one can see, a significant anti-correlation is observed yielding the correlation coefficient equal to $\mathrm{R}=-0.93$ and the regression coefficient equal to $\sim(6.4 \pm 0.6) \% / \mathrm{mm} \mathrm{Hg}$. Therefore, the resultant negative barometric coefficient at this period is a factor of 6.4 larger than that for cosmic ray hadrons at the Earth's surface being equal to $\sim-1 \% / \mathrm{mm} \mathrm{Hg}$.

\subsection{Response to Nepal's 2015 earthquake}

During the strong Nepal earthquake of magnitude 7.8 in April 2015 the PRISMA-YBJ array was running at a distance of $450 \mathrm{~km}$ from the epicenter. It is known that radon could be a precursor of nearest earthquake due to its long ( $3.8 \mathrm{~d}$ ) life-time when it can migrate to long distances in both air and in soil. Therefore, our method based on the recording of radon-due thermal neutrons makes it possible 


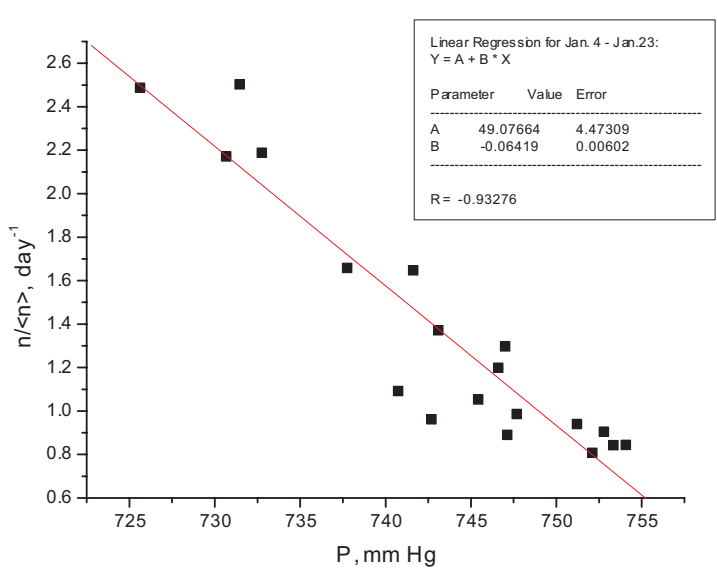

Figure 4. Anti-correlation plot between absolute 2-days delayed daily air pressure and normalized daily neutron counting rate for the dates Jan. 4-Jan. 232014.

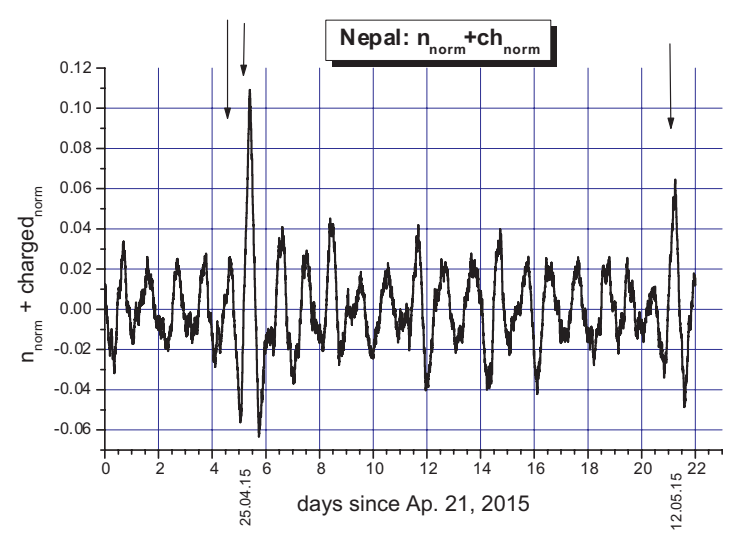

Figure 5. Response of the PRISMA-YBJ detectors to the Nepal earthquakes in April-May 2015.

to monitor soil radon concentration and thus to detect its change during earthquakes. For this purpose we use both of our counting rates: for thermal neutrons and for "charged". The latter was found to be sensitive to radon decays in air, while neutrons are sensitive to radon decays in soil [3].
Results are shown in Fig. 5 where the sum $\left(\sum\right)$ of normalized "neutrons" and "charged" is plotted. We use such a function to emphasize the change in phase of the daily time series of neutrons. It was mentioned above (see Fig. 1) that normally these two branches have anticorrelated phases of daily waves. But, during the days with a nearby strong earthquake, the phases coincide, the two waves do not compensate each other and $\sum$ has a maximum seen in Fig. 1.

It is noticeable that the effect we saw appeared not on April 25 when the strongest earthquake occurred $(\mathrm{M}=7.8)$, but only the next day (April 26) when an aftershock quake with $\mathrm{M}=6.7$ was observed. Two weeks later, another strong aftershock was observed and again we saw a significant peak of $\sim 6 \%$ amplitude. We can add that such high amplitude peaks were not observed during the whole period of data taking.

\section{Conclusion}

Using the en-detectors developed for EAS studies of variations of the environmental neutron flux, we have observed some geophysical phenomena very unusual for high-energy cosmic ray studies. We hope that the use of nuclear physics methods applied to geophysics can give novel interesting and unexpected results.

This work was supported in Russia by RFBR (grants 14-0200996, 16-32-00054 and 16-29-13067_ofi_m), RAS Presidium Program 'Fundamental properties of matter and astrophysics', and in China by NSFC (No. 10975046, No. 11375052).

\section{References}

[1] Yu.V. Stenkin, Nucl. Phys. B (Proc. Suppl.) 196, 293-296 (2009)

[2] B. Bartoli et al., Astropart. Phys. 81, 49-60 (2016)

[3] Yu.V. Stenkin et al. Pure and Appl. Geophys, in press; arXiv: 1605. 01283 [physics.geo-ph]

[4] S.N. Vernov et al. Proc. 16th Intern. Cosmic Ray Conf, 6, 129-134 (1979) 